\title{
Cognição e aprendizagem de L2: o que nos diz a pesquisa nos paradigmas simbólico e conexionista
}

\author{
Mailce Mota \\ Universidade Federal de Santa Catarina \\ Márcia Cristina Zimmer \\ UniRitter
}

\begin{abstract}
Este artigo apresenta os principais aspectos teóricos de dois paradigmas de investigação da cognição humana - o simbólico e o conexionista - e discute como a pesquisa em cada paradigma tem fomentado os estudos sobre aprendizagem de L2. O paradigma simbólico postula a existência de representações mentais através de regras lógicas e símbolos para explicar a aquisição e a utilização do conhecimento. O paradigma conexionista parte da premissa de que o processamento cognitivo está relacionado à maneira pela qual os neurônios se interconectam no cérebro. A partir dessas duas abordagens, o artigo examina como construtos tais como tipos de conhecimento, tipos de memória, processamento do insumo e atenção são apresentados nas duas teorias e como vêm sendo utilizados na pesquisa em L2.
\end{abstract}

This paper presents the main tenets of two paradigms of investigation of human cognition - the symbolic and the connectionist - and discusses how research in each paradigm has fed studies in L2 learning. The symbolic paradigm explains the acquisition and use of knowledge through the existence of mental representations, logical rules, and symbols. The connectionist paradigm claims that human cognitive processing is related to how neurons are interconnected in the brain. The paper also examines how constructs such as types of knowledge, types of memory, input processing, and attention are presented in the two theories and how these constructs relate to current L2 research.

\section{Introdução}

Como afirmado por Eysenck (1994, p. 66), é difícil indicar com exatidão o início do estudo sistemático de como os seres humanos aprendem e organizam o conhecimento. Entretanto, pode-se apontar Wilhelm Wundt como um dos precursores da psicologia experimental (LEAHEY, 1987, p. 182-183). Wundt é historicamente importante por 
ter apresentado a idéia de que as hipóteses sobre o processamento cognitivo deveriam ser submetidas a testes empíricos, trazendo, desta forma, o método experimental à psicologia. Preconizando o que começava a se firmar como ciência, Wundt fundou, em 1875 em Leipzig, o primeiro laboratório de psicologia experimental, onde conduziu estudos sobre a introspecção (KANDEL, SCHWARTZ e JESSEL, 1995, p. 322).

Observa-se, com base na trajetória dos estudos sobre a cognição humana, que na ciência cognitiva esse termo é utilizado para caracterizar os processos mentais envolvidos na percepção, apreensão e sistematização dos estímulos aos quais os indivíduos são expostos (ASHCRAFT, 1994; HABERLANDT, 1994; STERNBERG, 2003). Na psicologia cognitiva contemporânea, esses processos mentais são estudados a partir de dois paradigmas principais, o simbólico e o conexionista, que procuram explicar a construção do conhecimento. Esses paradigmas têm gerado estudos frutíferos em diversas áreas da ciência, sendo uma delas a aprendizagem de língua estrangeira/ segunda língua (doravante L2). ${ }^{1}$

A partir da dicotomia entre os paradigmas simbólico e conexionista, apresentamos, neste artigo, alguns dos construtos que têm se mostrado relevantes na pesquisa atual em L2. Na primeira parte, apresentamos o paradigma simbólico, descrevendo a teoria do processamento da informação. Na segunda parte, caracterizamos o paradigma conexionista, mostrando como o processamento cognitivo é visto neste referencial teórico. Em ambas as seções, construtos tais como tipos de conhecimento, tipos de memória e atenção são apresentados e suas aplicações na pesquisa em L2 são discutidas.

\section{O paradigma simbólico}

O paradigma simbólico postula estados mentais representacionais que se dão num nível simbólico e abstrato, bem como a existência de

\footnotetext{
${ }^{1}$ Neste trabalho não se adota a distinção, feita por Krashen (1982), entre os termos 'aquisição' e 'aprendizagem', seja em língua materna ou estrangeira. Esses termos serão usados de modo intercambiável, com o devido respaldo de Ellis (1994). Da mesma forma, os termos 'língua estrangeira' e 'segunda língua' serão tratados aqui como sinônimos.
} 
uma base de representação formal e lógica fundamental que descreve todos os objetos primitivos, as relações e as ações que compõem o mundo real (FODOR e PYLYSHYN, 1988). Assim, o conhecimento pode ser representado por regras lógicas e símbolos, e o comportamento inteligente advém da execução dessas regras e da manipulação desses símbolos (TORSUN, 1995). Fodor e Pylyshyn (1988) acrescentam, ainda, que a informação é representada por feixes de símbolos, que são produzidos em seqüência, de acordo com as instruções de um programa computacional simbólico. Dessa forma, todo o processamento das informações é feito em série.

A Inteligência Artificial (IA) surgiu no bojo desse paradigma cognitivo na década de 50, com o desenvolvimento da ciência da computação (HAYKIN, 1994, p. 3-5; TORSUN, 1995). Teóricos da IA postulavam que qualquer pensamento inteligente pode ter sua raiz nas computações de sistemas simbólico-físicos (CLARK, 1989). No modelo simbólico, o processamento de informação consiste essencialmente na transformação de símbolos de acordo com regras, que, na Inteligência Artificial, estariam estabelecidas num programa computacional (NEWELL, ROSENBLOOM e LAIRD, 1998, p. 96).

Nesse paradigma, há uma grande preocupação com a descrição do processo de representação, visto como uma manipulação de símbolos feita no nível semântico. Assim, a ênfase é dada aos processos mentais - softwares- sem preocupação alguma com a relação desses com a estrutura onde ocorrem: o cérebro - ou hardware. Esse paradigma está em consonância com a posição racionalista, ou platônico-cartesiana, segundo a qual a realidade mental é distinta da realidade física (DAMASIO, 2000). Essa visão dualista estabelece sua opção pela mente e pelo tratamento formal do símbolo, deixando o cérebro de lado.

Ao privilegiar a mente, centrando seus estudos sobre a cognição em representações mentais abstratas, o paradigma simbólico prioriza a construção de modelos de estruturas cognitivas de armazenamento e organização de conhecimento semelhantes a arquivos de um computador, como os esquemas e os frames (MINSKY, 1981).

A principal teoria do paradigma simbólico é a do processamento da informação, que compreende a cognição como a operação coordenada de vários processos mentais que se realizam em um sistema de memória multicomponencial (ASHCRAFT, 1994). A teoria do 
processamento da informação concebe os seres humanos como processadores da informação autônomos, ativos e de capacidade limitada, que possuem um sistema de memória composto de pelo menos quatro sistemas: a memória sensorial, a memória de trabalho, a memória de curto prazo e a memória de longo prazo (ASHCRAFT, 1994, p. 35). Ao procurar entender e explicar os processos mentais, os pesquisadores da teoria do processamento da informação enfocam a questão de como o conhecimento é representado, estruturado e processado na mente (ASHCRAFT, 1994). Para investigar esse assunto, são criados construtos tais como os quatro sistemas de memória, conhecimentos do tipo procedimental e declarativo, processamento automático e serial e atenção. Esses construtos constituem o foco da próxima seção.

\section{Memória, tipos de conhecimento, processamento e atenção}

A evidência para a existência de sistemas de memórias distintos foi primeiramente apresentada por Brown (1958) e Peterson e Peterson (1959), que demonstraram que a informação é rapidamente esquecida se não for de alguma forma manipulada através de processos cognitivos. Essa descoberta deu origem a vários estudos que mostraram que certos tipos de tarefa refletiam os processos de um sistema de memória de curto prazo e de capacidade limitada, distinto do sistema responsável pela retenção de conhecimento a longo prazo (CONRAD e HULL, 1964; BADDELEY, 1966a, 1966b; BADDELEY e WARRINGTON, 1970). Foi, entretanto, com Atkinson e Shiffrin (1968) que a distinção entre memória de longo prazo e memória de curto prazo se tornou clássica $\mathrm{e}$, conseqüentemente, fundamental para a teoria do processamento da informação (CANTOR e ENGLE, 1993; ERICSSON e KINTCH, 1995).

Ao longo dos últimos 40 anos, uma quantidade impressionante de experimentos foram conduzidos na tentativa de conceituar a memória de longo prazo, sendo três as principais abordagens de estudo desse tipo de memória. Na primeira, a memória de longo prazo é subdividida em memória episódica e memória semântica, uma distinção introduzida por Tulving $(1972,1985,2000)$. Em linhas gerais, a memória episódica armazena informações adquiridas através de experiências autobiográficas, ao passo que a memória semântica é responsável pelo armazenamento de informação enciclopédica (BOWER, 2000, p. 22-23). 
). Na segunda abordagem, a memória de longo prazo é subdividida em memória declarativa e procedimental, uma distinção embasada no trabalho do filósofo Ryle (1949), que foi o primeiro a observar que o conhecimento humano pode ser categorizado como sendo sobre as coisas e sobre como fazer as coisas. A memória declarativa é factual e caracteriza-se como um sistema que retém conhecimento explícito, acessível à consciência e verbalizável (HABERLANDT, 1994). Em contraste, a memória procedimental contém conhecimento implícito, que não está disponível à consciência e, portanto, não pode ser verbalizado. O conhecimento procedimental pode ser recuperado e utilizado de forma muito mais rápida do que o conhecimento declarativo. Finalmente, na terceira abordagem, a memória de longo prazo é concebida em termos de memória explícita e implícita, uma distinção originada na pesquisa em neuropsicologia (GRAF e SCHACTER, 1985, 1987). A memória explícita consiste de conhecimentos que podem ser conscientemente recuperados (GRAF e SCHACTER, 1985, p. 501), enquanto a memória implícita consiste de conhecimentos que, quando usados, não permitem a experiência consciente do uso desse conhecimento (SCHACTER, 1987).

O segundo maior sistema de memória humana, a memória de curto prazo, também foi alvo de ampla pesquisa, desenvolvida sobretudo nos anos 60 e 70. Entre os muitos modelos de memória de curto prazo que surgiram, o de Atkinson e Shiffrin (1968) veio a ser o mais influente: incluía a memória sensorial, a de curto prazo e a de longo prazo, sendo o sistema de curto prazo o componente mais importante e mais estudado. Para Atkinson e Shiffrin (1968), essa memória era um sistema unitário de capacidade limitada, necessária tanto para a aquisição como para a utilização da informação. Para eles, a memória de curto prazo poderia estocar a informação através do processo de rebearsal, o que aumentava a probabilidade de transferência do conhecimento para a memória de longo prazo. Embora estivessem conscientes da existência e da relevância dos aspectos semânticos do material a ser armazenado, Atkinson e Shiffrin (1968) tinham como interesse principal rote rehearsal, mecanismo que consistia da repetição pura.

Apesar de ser capaz de explicar uma variedade de dados, o modelo de Atkinson e Shiffrin era particularmente deficiente em 
explicar o comportamento de pacientes com problemas de memória de curto prazo que não apresentavam problemas na memória de longo prazo. A hipótese de que quanto mais tempo o material fosse mantido na memória de curto prazo, maior a possibilidade de ser transferido para a memória de longo prazo não foi corroborada por um grande número de estudos que mostravam que a simples repetição não levava necessariamente à aprendizagem.

Percebendo esse e outros problemas no modelo, Baddeley e Hitch (1974) propuseram um modelo de memória de curto prazo multicomponencial e dinâmico, que foi chamado de memória de trabalho e considerado responsável pelo armazenamento e processamento simultâneos da informação. A diferença crucial ente o modelo de Baddeley e Hitch e o modelo de Atkinson e Shiffrin é que o primeiro possui três componentes: a) um executivo central, semelhante ao mecanismo da atenção, responsável pela seleção e controle de vários processos de nível superior, tais como o raciocínio, a organização e a recuperação de informações; b) um circuito articulatório (phonological loop), que serviria para reter informações sob a forma fonológica (através de repetição subvocal); c) uma tábua visuo-espacial (visuospatial scratch pad), encarregada da codificação de informações visuais e espaciais. O mais importante, contudo, é que Baddeley e Hitch enfatizaram a eficiência do sistema, que seria capaz de lidar com diferentes tipos de informação, o que lhe conferiu dinamicidade.

Depois da proposta de Baddeley e Hitch, um novo paradigma de pesquisas sobre memória humana foi estabelecido, de forma que a memória de curto prazo é atualmente compreendida como um fragmento de um sistema mais potente, a memória de trabalho, responsável pelo processamento e pela coordenação dos processos mentais, em tempo (BADDELEY, 2000, p. 77). Três décadas de pesquisa sobre a memória de trabalho resultaram em uma enorme quantidade de estudos, mais de 10 modelos diferentes e diversas definições do conceito (SHAH e MIYAKE, 1999, p. 2; MIYAKE e SHAH, 1999, p. 443).

Há duas abordagens principais de estudo da memória de trabalho. A primeira utiliza dados neuropsicológicos de pacientes com lesões cerebrais para determinar a estrutura do construto (BADDELEY, 1992). A segunda abordagem aplica correlações psicométricas e enfatiza a relação entre diferenças individuais na capacidade da memória de 
trabalho e o desempenho cognitivo em tarefas complexas (BADDELEY, 1992). Dentro desta abordagem, Engle, Kane e Tuholsky (1999) propõem que a capacidade da memória de trabalho seja operacionalizada como "a capacidade para controlar e sustentar a atenção mediante a interferência e a distração" (p.104). ${ }^{2}$ Nesse sentido, o conceito de atenção surge como fundamental na teoria do processamento da informação, embora ainda seja objeto de controvérsia nas ciências cognitivas.

Definida de forma ampla, a atenção é um recurso mental limitado, seletivo e sujeito ao controle voluntário dos indivíduos (GOPHER, 1994, p. 23-28). Esse mecanismo cognitivo permite o acesso da informação à consciência e é essencial ao controle da ação. O grau de atenção dispensado ao desempenho de determinadas tarefas define o quanto os processos nelas envolvidos estão ou não automatizados. Derivam daí dois conceitos de processamento cognitivo: os processos automáticos e os processos controlados. Os automáticos não exigem o processamento de recursos, liberando assim o sistema cognitivo para aspectos mais complexos da tarefa. Já que não demandam muitos recursos atencionais, os processos automáticos são altamente eficientes e podem ser executados em paralelo. Os processos controlados, por outro lado, necessitam dos recursos atencionais e são executados de forma serial. Por fim, cabe frisar que, para vários pesquisadores (ROBINSON, 2001, por exemplo), a atenção é essencial para que a aprendizagem ocorra.

Os vários construtos aqui descritos têm sido utilizados na pesquisa em L2 de diversas maneiras, originando várias hipóteses acerca do papel desses construtos na aprendizagem da L2. Essa é a temática da próxima seção.

\section{O papel dos construtos cognitivos na pesquisa em L2: a visão simbólica}

A visão da psicologia cognitiva, com a conseguinte aplicação de seus construtos, tem sido utilizada na pesquisa em L2 em quatro

\footnotetext{
${ }^{2}$ No original, grifado: "the capacity for controlled, sustained attention in the face of interference or distraction."
} 
principais linhas: a Noticing hypothesis (SCHMIDT, 1990, 1995, 2001), o processamento do insumo lingüístico (VANPATTEN, 1990, 1996; VANPATTEN e CADIERNO, 1993; VANPATTEN e SANZ, 1995), a hipótese da produção (the Output bypothesis) (SWAIN, 1985, 1995) e memória de trabalho (HARRINGTON, 1992; HARRINGTON e SAWYER, 1992; MIYAKE e FRIEDMAN, 1998).

Uma linha de pesquisa em L2 elaborada a partir do construto da atenção é aquela proposta por Schmidt (1990, 1995, 2001) e por ele denominada de Noticing hypothesis. Partindo do pressuposto de que a capacidade humana de processamento da informação é limitada e de que a atenção é condição imprescindível para a aprendizagem, essa hipótese postula que somente aqueles aspectos do insumo que recebem a atenção focal do aprendiz é que são incorporados à interlíngua. Em sua proposta, Schmidt afirma que a consciência no nível do notar/ reparar, por parte do aprendiz, no momento da aprendizagem é condição necessária e suficiente para que o insumo se torne intake. A Noticing hypothesis tem motivado vários estudos sobre a relação entre o processo cognitivo de notar/reparar aspectos da L2 e mudanças na interlíngua. Assim, a partir dessa proposta, Leow (1997) investigou a relação entre o grau de atenção e a aquisição de estruturas gramaticais em espanhol como L2. A análise qualitativa e quantitativa dos resultados revelou que diferentes graus de consciência ${ }^{3}$ levam a diferentes tipos de processamento. Além disso, Leow apontou que um grau de atenção maior leva a um reconhecimento melhor e a uma produção escrita mais precisa das estruturas gramaticais e que esses resultados oferecem evidência empírica para o papel facilitador da atenção na aprendizagem de L2.

Certamente, uma das linhas de pesquisa em L2 mais profícuas na utilização de construtos cognitivos como a atenção é a do processamento do insumo, apresentada por VanPatten $(1996,2004)$ em seu modelo de aquisição de L2. Partindo uma vez mais da noção fundamental de que possuímos uma capacidade limitada de processamento da informação, VanPatten (2004) afirma que aprendizes de L2 apresentam dificuldade

\footnotetext{
${ }^{3} \mathrm{O}$ termo usado por Leow é levels of awareness. O acesso à consciência é um elemento chave que inicia com a percepção do estímulo, é notada e vai gradativamente aumentando até chegar à consciência plena.
} 
em focalizar a atenção em todos os aspectos do insumo lingüístico, o que os faz selecionar itens específicos e processar os outros componentes de forma periférica. Nesse modelo, a atenção é um pré-requisito para que a aprendizagem aconteça. Nesse sentido, VanPatten (2004) postula que a atenção do aprendiz deve ser dirigida para aquelas partes do insumo que são relevantes para a tarefa em questão. Interessado na aplicação pedagógica de seu modelo, VanPatten e colaboradores desenvolveram uma série de estudos sobre uma técnica instrucional conhecida como a instrução sobre o processamento (processing instruction), cujo objetivo é facilitar mudanças na interlíngua a partir da manipulação da atenção do aprendiz sobre o insumo lingüístico (VANPATTEN E CADIERNO, 1993; VANPATTEN, 2004).

A terceira linha de pesquisa em L2 que vem utilizando os construtos cognitivos é aquela referente à hipótese da produção (SWAIN, 1985), que propõe que a produção oral e a escrita desempenham um papel ativo na aprendizagem de L2, uma vez que essas atividades de produção são vistas como catalisadoras do desenvolvimento da interlíngua. Segundo Swain (1995, p. 125-126), a produção (oral e/ou escrita) tem quatro funções principais na aquisição da L2: 1) desenvolver no aluno a automaticidade e a fluência no uso da L2; 2) fazer o aprendiz focalizar sua atenção em aspectos gramaticais da língua; 3) auxiliar o aprendiz na criação de hipóteses sobre a L2; 4) estimular o aprendiz a desenvolver sua capacidade metalingüística. Dessas quatro funções, a que mais tem atraído o interesse de pesquisadores é a segunda, uma vez que o principal processo cognitivo nela envolvido é a atenção (SWAIN e LAPKIN, 1994; SWAIN, 1995; IZUMI, 2002) - ou seja, essa função pressupõe que o aprendiz, ao tentar expressar uma idéia na L2, percebe as lacunas no seu conhecimento da interlíngua, o que vai levá-lo a procurar maneiras de vencer suas dificuldades lingüísticas. Nessa procura, o aprendiz engaja-se em vários processos mentais que vão consolidar o conhecimento da L2 que já possui ou gerar novo conhecimento. Em ambos os casos, a aprendizagem é estimulada a partir da atenção que o aprendiz dá às estruturas gramaticais de que necessita para se expressar.

$\mathrm{Na}$ investigação dos aspectos psicolingüísticos envolvidos na hipótese da produção, Izumi e colaboradores (IZUMI e BIGELOW, 2000; IZUMI, 2002) investigaram o papel da atenção durante a produção 
de aprendizes de diferentes línguas estrangeiras. Os resultados mostraram que as oportunidades contínuas de produzir a L2 utilizando as estruturas lingüísticas em foco são cruciais para o aprimoramento do uso dessas estruturas por parte dos aprendizes, além de serem mais eficazes do que as práticas que envolvem apenas atividades de compreensão do insumo. As pesquisadoras apontam, entretanto, que a produção da língua nem sempre levou o aluno a focalizar sua atenção nas estruturasalvo, o que pode ser explicado tanto por fatores individuais como pela natureza das estruturas (IZUMI, 2002).

No que diz respeito aos tipos de memória, a pesquisa em L2 tem destacado a memória de trabalho e suas relações com a compreensão leitora, a aquisição e compreensão da sintaxe e a produção oral. Harrington (1992) investigou até que ponto o conhecimento de vocabulário e gramática afeta a relação entre a capacidade de memória de trabalho e a compreensão de texto em L2. Os resultados demonstraram que há uma correlação significativa entre a capacidade da memória de trabalho, o conhecimento gramatical, o conhecimento de vocabulário e a compreensão de textos, replicando, dessa forma, os resultados da pesquisa em L1. Os resultados de Harrington (1992) foram repetidos por Harrington e Sawyer (1992). Em particular, esses pesquisadores demonstraram que não há diferença significativa entre a capacidade de memória de trabalho na L1 e na L2. Em consonância com esses resultados, Berquist (1998) mostra que a capacidade da memória de trabalho em L2 é um importante elemento de predição da proficiência na L2. Já Miyake e Friedman (1998) investigaram a relação entre a capacidade da memória de trabalho e a aquisição e compreeensão de estruturas complexas na L2. Os resultados mostraram que os aprendizes com maior capacidade de memória de trabalho eram mais eficientes na tarefa de compreender e aprender essas estruturas.

Por fim, no Brasil a pesquisa sobre memória de trabalho na L2 tem-se mostrado particularmente frutífera. Fortkamp (1999, 2000, no prelo), por exemplo, encontrou uma relação significativa entre a capacidade de memória de trabalho e a produção oral em L2, demonstrando que indivíduos com maior capacidade são mais fluentes e mais precisos no domínio da estrutura léxico-gramatical e utilizam estruturas mais complexas e vocabulário mais sofisticado quando se expressam oralmente na L2. Mendonça (2002) demonstra que 
aprendizes com maior capacidade de memória de trabalho utilizam maior número de estratégias de aprendizagem de vocabulário da L2. Torres (2003) encontrou uma relação significativa entre a capacidade de memória de trabalho e a identificação de idéias principais em textos de L2. Em geral, esses estudos apresentam resultados consistentes, sugerindo que a capacidade da memória de trabalho tem um papel fundamental no desempenho de tarefas em L2.

\section{O paradigma conexionista}

O conexionismo, auxiliado pela simulação em computador das redes neuroniais, vem provocando grande debate dentro da Psicologia Cognitiva e da Lingüística, ao partir da premissa de que o processamento cognitivo está intimamente ligado à maneira como os neurônios se interconectam no cérebro. Dessa forma, processos cognitivos como a memória e a aprendizagem são estudados levando em conta sua base física e o meio ambiente onde se situa o sistema em que eles ocorrem. Além disso, o conexionismo explora uma importante característica do funcionamento cognitivo humano, que é a capacidade de produzir generalizações espontâneas, ou seja, fazer generalizações a partir de experiências específicas, o que dá conta das diferenças individuais no desenvolvimento e na aprendizagem de mecanismos cognitivos como a L2 (BROEDER e PLUNKETT, 1994).

De acordo com Shanks (1993), a idéia central do conexionismo está no processamento de informação do cérebro, que se dá através de redes neuroniais - cujas células nervosas, os neurônios, comunicam-se umas com as outras através da transmissão de impulsos elétricos. Um princípio básico do conexionismo é que a maioria das redes neuroniais do cérebro, senão todas, passa por mudanças sutis que ocorrem nas sinapses entre os neurônios. Ao estimular certos subconjuntos de neurônios com mais freqüência do que outros, as experiências de mundo dos indivíduos vão reforçando certas sinapses e tornando alguns padrões de atividades elétricas mais fortemente impressos do que outros. Esses padrões formariam a base da memória e do aprendizado. As sinapses, responsáveis pelo processamento das informações (como por exemplo, input auditivo ou visual), acontecem simultaneamente em diferentes redes neuroniais localizadas em partes diferentes do 
cérebro; daí a denominação desse tipo de funcionamento como processamento de distribuição em paralelo ou PDP (RUMELHART e MCCLELLAND, 1986).

No que tange à simulação computacional da cognição em geral, o conexionismo surgiu como uma reação contra os modelos simbólicos estipulativos - sistemas que modelavam a cognição, estipulando a existência de um conjunto correto ou ideal de regras em termos de seus elementos, combinações e padrões de ordenamento de regras. Atribuise o sucesso desses sistemas estipulativos à precisão de sua formulação e à expressividade da produção formal do sistema lingüístico no qual se baseavam (MacWHINNEY, 2002). Contudo, à medida que esses sistemas cresciam em complexidade, tornou-se impossível testar a plausibilidade empírica de seus componentes. Adotando uma formulação emergentista da base neuronial da cognição, o conexionismo oferece um arcabouço teórico em que as estruturas emergem da interação entre processos biológicos e ambientais. McClelland et al. (2003) discutem a diferença entre a abordagem simbólica dos modelos estipulativos incluindo modelos híbridos como ACT-R (ANDERSON et al., 1998), que incorpora à sua arquitetura alguns princípios conexionistas - e a conexionista, apontando as principais diferenças entre os modelos computacionais implementados por esses dois paradigmas.

A primeira distinção refere-se à ênfase na arquitetura - adotada pelos modelos simbólicos - e à ênfase nos princípios, refletida nos modelos conexionistas. Enquanto os sistemas de natureza estipulativa - como os desenvolvidos por Newell (1980) e Anderson et al. (1998) - têm como objetivo principal criar uma única arquitetura cognitiva que possa desempenhar qualquer tarefa, a abordagem PDP tenta elucidar os princípios gerais que governam todos os aspectos da cognição. Dessa forma, os modelos simbólicos tendem a reificar seus princípios na arquitetura, que se torna demasiadamente complexa e reflete os princípios baseados na serialidade dos processos cognitivos, ao passo que os pesquisadores conexionistas buscam, através de modelos e simulações extremamente simples, capturar o conjunto de princípios gerais sobre a natureza da aprendizagem e do processamento no sistema cognitivo humano.

McClelland et al. (2003) afirmam que a busca pelos princípios gerais (domain general) subjacentes a diferentes domínios cognitivos, 
como a linguagem e a visão, implica um tipo de investigação que não os estude como módulos cognitivos distintos que funcionam de acordo com princípios específicos. Dessa forma, enquanto muitos trabalhos simbólicos tentam elucidar os princípios específicos de vários domínios, tal como Chomsky, na linguagem, e Marr, na visão, os investigadores conexionistas acreditam que os estudos detalhados de alguns fenômenos específicos devem buscar a compreensão dos princípios gerais da cognição. Entram em cena, então, as simulações, que funcionam como uma maneira de implementar a teoria, instanciando os princípios teóricos do paradigma. Dessa forma, o ciclo composto pelas etapas de construção de modelos simples, experimentação e reestruturação de modelos leva a um maior esclarecimento dos princípios gerais subjacentes a cada simulação específica. Segundo Plaut (2003), os modelos e suas arquiteturas nunca estão completamente corretos - já que eles geralmente falham em alguns detalhes apresentados nos comportamentos que buscam replicar -, mas a teoria subjacente a eles deve buscar a explicação e a correção.

Outra distinção enumerada por McClelland et al. (2003) diz respeito à natureza e ao papel do nível simbólico. Enquanto os estudiosos da visão simbólica asseveram que os princípios fundamentais a nortearem a cognição podem ser especificados por meio da estipulação e manipulação de símbolos, os conexionistas afirmam que os princípios ${ }^{4}$ que governam a cognição são aqueles subjacentes aos processos que ocorrem em unidades neuroniais simples e suas conexões. Isso significa que a abordagem conexionista não nega a existência de um nível simbólico de pensamento, mas sim a importância desse nível. Seus modelos refletem uma teoria explanatória na qual regras e símbolos não são necessários nem norteiam o funcionamento cognitivo. McClelland

\footnotetext{
${ }^{4}$ Tais princípios foram explicitados por Plaut et al. (1996, p. 59) através da acronímia GRAIN: Graded (gradual e contínuo; refere-se tanto à propagação da ativação nas redes como à aprendizagem na maioria dos domínios); Random (refere-se ao fato de que as ativações das unidades neuroniais estão sujeitas à variabilidade estocástica); Adaptive (relaciona-se ao fato de que o ajuste de pesos nas conexões entre as unidades melhora o desempenho da rede); Interactive (explicita a interação entre camadas de unidades neuroniais e entre redes); Non-linear (descreve o output como funções não-lineares de todas as unidades de input. Acrescente-se a essas propriedades o princípio da distribuição da informação em paralelo.
} 
(comunicação pessoal) afirma que a abordagem conexionista, tal como vista por ele e Rumelhart, busca "desempacotar essa superestrutura denominada símbolo", destacando a emergência de fenômenos cognitivos complexos - como a linguagem - originados no nível atômico por unidades simples.

No que concerne à linguagem, então, a visão emergentista adotada pelo conexionismo substitui o confronto entre visões radicais da aquisição da linguagem, como o inatismo e o empiricismo, por um novo corpo teórico, evitando "as armadilhas do pensamento dicotômico na área dos estudos da linguagem e da cognição" (ALBANO, 1999, p. 50). Esse novo cabedal teórico foi explicitamente elaborado para dar conta, em termos de mecanismos, das interações entre processos cognitivos, descartando distinções entre conhecimento e processamento lingüístico, competência e desempenho. A linguagem é fruto do entrelaçamento de diversos tipos de processamentos de informações advindas de vários sistemas - auditivo, motor, visual, articulatório - e em vários níveis - do genético ao neuronial. Daí a afirmação de que o conhecimento lingüístico não é inato, mas emergente (ELLIS, 1999). De acordo com MacWhinney (2002), é importante que o emergentismo faça referência ao corpo, pois assim o estudo da aquisição e do processamento da linguagem leva em consideração contextos interativos como o trato fono-articulatório, o cérebro e o meio em que está inserido o falante.

A orientação da abordagem conexionista pelo desempenho funda-se no pressuposto chave de que o estímulo lingüístico é rico o suficiente para que o aprendiz capture as regularidades do sistema lingüístico. De acordo com essa visão: a) o ambiente lingüístico apresenta muitas regularidades distribucionais que norteiam a aprendizagem da linguagem; b) a aquisição da linguagem envolve a exploração das restrições probabilísticas existentes em vários tipos de informação lingüística e não lingüística; c) não há uma divisão estrita entre conhecimento lingüístico e extra-lingüístico: a eficácia da aprendizagem depende tanto da estrutura do insumo quanto do conhecimento prévio (lingüístico e de mundo); d) a informação distribucional pode fornecer evidência negativa implícita (RHODE e PLAUT, 2003; SEIDENBERG e McDONALD, 1999). Alguns desses princípios têm encontrado eco na noção de aprendizagem estatística da linguagem, noção essa já estudada por Harris (1955) na tradição 
estruturalista-distribucionalista e recentemente resgatada por Saffran (SAFFRAN, 2001; SEIDENBERG, MACDONALD e SAFFRAN, 2002).

Ao enfatizar o processo de aprendizagem, mostrando a sensibilidade dos processos cognitivos ao contexto, o conexionismo resgata, nos estudos da aquisição da linguagem, a importância do desenvolvimento e da mudança gradual operada à medida que a língua estrangeira vai sendo apreendida. Ellis (1999, p. 35), por exemplo, afirma que a abordagem funcionalista da linguagem é a que mais se presta para o estudo da aprendizagem da L2 de um ponto de vista conexionista, uma vez que o funcionalismo enfatiza o processo de desenvolvimento e a interação, e a aprendizagem lingüística assemelha-se a outras habilidades complexas cujo desenvolvimento demanda muito tempo.

A aprendizagem da língua estrangeira é um processo longo e complexo. Embora longo e complexo como o da língua materna, o processo de desenvolvimento da língua estrangeira difere do da língua materna de várias maneiras, e uma delas diz respeito à transferência dos padrões lingüísticos da L1 para a L2. Segundo Gasser (1990), o fenômeno cognitivo em que os modelos conexionistas são extraordinariamente hábeis em simular é o da transferência de conhecimento - lingüístico ou extralingüístico.

Tendo em vista as principais propostas conexionistas em relação à cognição e à aprendizagem da língua estrangeira, pode-se discutir como o conexionismo aborda, na pesquisa em L2, construtos como memória, tipos de conhecimento e processamento do insumo.

\section{O papel dos construtos cognitivos na pesquisa em L2: a visão conexionista}

Partindo da premissa de que o significado se forma a partir de complexos padrões de atividades entre os neurônios, que são responsáveis, em última instância, pela formação do aprendizado e da memória via processamento distribuído em paralelo, o conexionismo modificou radicalmente a noção que se tinha sobre a memória em geral e a memória de trabalho em particular, que passa a ser vista como um mecanismo de ativação de processamento da memória perene (SOUGNÉ e FRENCH, 1997), dispensando noções como armazenadores e tornando obsoletas divisões como as existentes entre memória de 
trabalho e memória de longo prazo. Na visão conexionista, a memória de trabalho corresponderia ao tempo durante o qual um determinado padrão elétrico estaria ativado, mediante um insumo que, no caso da leitura, seriam as letras ou frases de um texto. Além disso, propriedades como a distribuição da informação em paralelo e o controle distribuído da informação contribuem para a diluição da diferença entre a noção de conhecimento e a de processamento: "Não há, no cérebro, salas de gravação nem lugares de armazenamento separados dos mecanismos que computam a informação. A distinção estrita entre processamento e conhecimento fica diluída". ${ }^{5}$ (ELMAN et al., 1996, p. 314-315)

No que tange às intermináveis discussões sobre as fontes de diferenças individuais entre o processamento da informação lingüística em leitores e falantes, tanto da L1 quanto da L2, MacDonald e Christiansen (2002) concordam com Elman et al., afirmando que essas se devem a dois fatores principais, que são o grau de experiência com a língua e fatores biológicos, como a velocidade do processamento cognitivo. Ao mencionarem a divisão existente entre a memória de trabalho e a memória de longo prazo nos modelos simbólicos tradicionais de leitura, esses pesquisadores fazem o seguinte comentário, que reitera a questão da indissociabilidade entre conhecimento e processamento (ELMAN et al., 1996), que parece estar ligada ao caráter distribuído da informação nos modelos conexionistas:

Os pesquisadores tradicionalmente postulam a existência de uma memória de trabalho para o processamento e o armazenamento temporário, separado da representação do conhecimento na memória de longo prazo. Em nosso ponto de vista, nem o 'conhecimento' nem a 'capacidade' são primitivos que possam ser manipulados independentemente na teoria ou em modelos computacionais. (...) Na nossa visão, o conhecimento lingüístico de longo prazo não é funcionalmente separado do locus do processamento. $^{6}$ (MACDONALD e CHRISTIANSEN, 2002, p. 38)

\footnotetext{
5 "There is no tape-room in the brain, no storage bins that are separate from the mechanisms that compute information. The strict distinction between processing and knowledge is blurred."

6 "Researchers postulated a working memory for temporary storage and processing, separated from the representation of long-term knowledge. On our account, the long-term knowledge of language is not functionally separated from the locus of processing."
} 
A última frase destaca, uma vez mais, a associação da estrutura - o locus do processamento - à função, mencionada na seção anterior. Percebe-se, a partir disso, que a abordagem conexionista não postula a existência de armazenadores separados de memória nem na L1 nem na L2, pois nem sequer admite a existência de divisão entre memória e processamento cognitivo. Em pesquisas conduzidas na Unicamp, Rinaldi (2003) e Rinaldi e Françozo (2004) construíram um modelo conexionista para a memória bilíngüe, investigando, através de simulações com redes conexionistas recorrentes simples, como se organiza o léxico mental bilíngüe. Seus resultados apontam para a existência de um único léxico mental.

Então, ao invés de entrar no debate sobre o papel de construtos como a memória de trabalho na aprendizagem da L2, os pesquisadores conexionistas preferem investigar como os aprendizes da L2 - ou as redes conexionistas - processam a informação lingüística, abordando conjuntamente construtos como memória, tipos de conhecimento e processamento do insumo na pesquisa em L2.

Esse é o caso do Modelo Hipcort (McCLELLAND, McNAUGHTON e O'REILY, 1995), que sugere que a aprendizagem e a memória emergem da interação entre o processamento de dois sistemas cognitivos complementares. De acordo com esse modelo, a memória e a aprendizagem são formadas por alterações sinápticas que ocorrem no sistema do hipocampo, cujo mecanismo de formação de memória é extremamente veloz. Essas sinapses podem ser reinstanciadas no neocórtex, um sistema de aprendizagem bastante lento e gradual. Dessa forma, a aprendizagem inicia-se no hipocampo e resulta na formação de um traço de memória que pode ser reativado de forma explícita. $\mathrm{O}$ neocórtex também auxilia na aprendizagem, mas opera lentamente através de pequenos incrementos nas forças de conexão entre suas sinapses para deslindar a estrutura complexa de conjuntos de experiências, auxiliando a associação de novos insumos com itens já codificados no córtex, que formam o conhecimento prévio. Essa aprendizagem poderia ser qualificada como implícita, pois advém de mudanças sinápticas pequenas demais para ensejar a ativação explícita do conhecimento.

É importante ressaltar que há uma interação entre os conhecimentos de ambos os sistemas, uma vez que o resultado desse processamento 
rápido, que se dá no hipocampo, pode ser integrado gradualmente ao sistema do neocórtex, não se tratando, dessa forma, de dois mecanismos de aprendizagem isolados um do outro, mas sim de dois sistemas efetivamente complementares, conforme ressaltam McClelland et al. (1995). Essa complementaridade entre o processamento dos dois sistemas se dá através do processo de consolidação, que possibilita que o conhecimento inicialmente gerado no hipocampo possa ser incorporado ao sistema do neocórtex através de reinstanciações sinápticas que seriam responsáveis pela engramação do novo conhecimento ao conhecimento prévio. Percebe-se, assim, existir uma interação gradiente entre a codificação explícita e a implícita na formação de novas memórias ou conhecimentos à medida que o processo de consolidação vai se desenrolando.

A transferência do conhecimento da L1 para a L2 poderia ser explicada, em termos de sistemas complementares, como a aprendizagem associativa que vai sendo consolidada no neocórtex. Quando o conhecimento prévio da L1 diverge das associações da L2 que estão sendo aprendidas no hipocampo, a participação do neocórtex pode levar à transferência do conhecimento da língua materna para a língua estrangeira. Nesse caso, o processamento no hipocampo tem que ser muito mais intenso e repetitivo, a fim de superar a ativação das associações desviantes advindas do córtex, onde o conhecimento da L1 está enraizado.

MacWhinney (2001) afirma que o fato de o cérebro ser estruturado de maneira a promover a transferência de informação neuronial tem conseqüências cruciais para a aprendizagem da L2. Tendo adquirido a língua materna na infância, o aprendiz já traz para a aprendizagem da língua estrangeira um sistema neurolingüístico muito bem organizado. Inicialmente, a aprendizagem da L2 é altamente influenciada pelas estruturas da língua materna, tanto no léxico como na fonologia (FLEGE, 2002; FLEGE e LIU, 2001). Ao construir ligações diretas entre sons e significados na L2 e ao reestruturar conceitos já existentes na língua materna, o aprendiz vai, pouco a pouco, aumentando o acesso automático ao léxico e à estrutura gramatical e fonológica na língua estrangeira sem recorrer à língua materna. Essa automaticidade forma uma barreira contra os efeitos da interferência da língua materna sobre a estrangeira. A reestruturação desfaz a forte associação inicial entre a 
língua materna e a estrangeira, embora algum grau de transferência entre as duas línguas esteja sempre presente, dada a natureza interativa do processamento cognitivo (MacWHINNEY, 2001).

Pesquisas conexionistas da aprendizagem da L2 abordam o papel da transferência do conhecimento da L1 para a L2 em áreas como a morfossintaxe - através de simulações da aprendizagem da ordem das palavras (word order) na frase (GASSER, 1990), da aquisição de pronomes do holandês por falantes nativos do turco (BROEDER e PLUNKETT, 1994) - e como a fonologia - através de simulações, baseadas na noção de aprendizagem hebbiana, ${ }^{7}$ sobre a percepção e produção do $/ \mathrm{l} / \mathrm{e} / \mathrm{r} /$ por falantes japoneses aprendizes de inglês (McCLELLAND, 2001).

O Modelo de Competição (BATES e MacWHINNEY, 1989; MacWHINNEY, 1997) é um exemplo de investigação conexionista que caracteriza a aquisição da L2 como sendo guiada por uma análise distribucional de pistas - ou propriedades salientes do insumo - no fluxo da fala. A teoria conexionista forneceu ao modelo de competição um instrumento de implementação para compreender como vários tipos de pistas podem interagir dentro de um quadro distribucional através do uso de modelos conexionistas simples que relacionam pistas lexicais (como a ordem das palavras, a morfologia da concordância verbal, a marcação de caso, etc.) com interpretações funcionalistas (como agente/paciente, topicalidade, perspectiva, etc.) na compreensão e produção de frases (KEMPE e MacWHINNEY, 1999). A parte de competição do modelo mostra como o uso da inferência bayesiana

\footnotetext{
${ }^{7}$ Segundo McClelland (2001), a regra de Hebb sugere que os mecanismos de modificação sináptica tendem a reforçar o padrão que um determinado input tenha ativado, pois estudos sobre a potenciação de longo prazo sugerem que quanto mais forte a ativação desencadeada por um determinado input, mais forte será o efeito e mais tempo ele durará. O resultado, então, é um aumento na probabilidade de que um input subseqüente e muito semelhante produza a mesma ativação. Se a ativação for adequada e útil, a aquisição e a manutenção das habilidades cognitivas desejáveis ocorrerá. Entretanto, se a ativação for inapropriada, o ajuste sináptico hebbiano tenderá a reforçar as tendências existentes, e não ocorrerá progresso na aquisição do efeito desejado. McClelland sustenta que a dificuldade em produzir uma fala sem sotaque na L2 pode advir de um reforço indesejável de ativações preexistentes relacionadas à fala em L1.
} 
(modelo estatístico usado para lidar com a interação de diversas variáveis) pode levar à ativação de uma única hipótese de interpretação dentro de uma rede rica em associações e conexões interagindo simultaneamente (algumas competindo e outras se reforçando mutuamente, como resultado das muitas redundâncias da língua). O modelo foi testado na avaliação das pistas e na predição dos efeitos da transferência lingüística, atuando como um substituto da análise contrastiva, mas com um viés probabilístico mais acentuado (MacWHINNEY, 1992).

No Brasil, Zimmer (2004a, 2004b) apresenta uma simulação em redes conexionistas do tipo feedforward sobre os processos de transferência do conhecimento fonético-fonológico do PB (L1) para o inglês norte-americano (L2) durante a leitura de palavras e não-palavras, abordando não apenas a transferência do conhecimento da L1 para a L2, mas também o papel desempenhado pelo insumo no processamento lingüístico. Com o objetivo de verificar se a incidência de tais processos varia em função do tipo de insumo recodificado (palavras regulares de alta freqüência, palavras regulares de baixa freqüência, palavrasexceção de alta freqüência, palavras-exceção de baixa freqüência e não-palavras), esse estudo coloca em evidência um elemento de destaque nos estudos conexionistas da L2: o interesse em descobrir como os aprendizes lidam com os efeitos conjuntos da freqüência e da regularidade do insumo lingüístico, que são subprodutos diretos da experiência, do conhecimento prévio dos aprendizes, do contato com a L2 (ELLIS, 2001; MacDONALD e CHRISTIANSEN, 2002).

Nos estudos de aquisição de língua estrangeira, ainda não se sabe até que ponto a quantidade e a qualidade do insumo determinam como a língua-alvo é percebida e processada pelo aprendiz, tornando-se intake, e como a quantidade e a qualidade do insumo afetam a proficiência na língua-alvo. Comparada à aquisição da língua materna, que se baseia principalmente em dados naturais, o insumo na aquisição da língua estrangeira varia significativamente de acordo com o ambiente de ensino/aprendizagem. Os resultados advindos de estudos empíricos e computacionais conexionistas em L2 no exterior (SOKOLIK, 1990; BROEDER e PLUNKETT, 1994; MacWHINNEY, 2001) e no Brasil (RINALDI, 2003; SIGOT, 2002; ZIMMER, 2004a, 2004b) indicam que a resposta a qualquer tipo de insumo é uma função da 
experiência do falante ou da rede conexionista. Isso se deve ao fato de os modelos conexionistas serem sensíveis à tensão entre o conhecimento genérico, derivado das sobreposições entre o que há de comum - consistente - entre os padrões presentes no insumo, e o conhecimento específico, advindo das idiossincrasias mais marcantes do mesmo. Essa fusão prototípico-exemplar de modelos de aprendizagem e formação de conceitos, aliada ao efeito da freqüência de exposição ao insumo que caracteriza a experiência do aprendiz (ou das redes conexionistas), é uma das mais interessantes propriedades da aprendizagem da $\mathrm{L} 2 \mathrm{em}$ redes conexionistas, que captam não somente as similaridades mais características do insumo, mas também as singularidades mais marcantes, as idiossincrasias presentes no mesmo. ${ }^{8}$

Como a freqüência de exposição ao insumo é muito importante na aprendizagem da L2, no conexionismo a instrução explícita é tida como um fator chave para a questão do monitoramento da aprendizagem e no desenvolvimento de habilidades metacognitivas, principalmente como tratamento pedagógico dos desvios fossilizados de produção e/ou percepção oral (McCLELLAND, 2001; ALVES, 2004). Mais uma vez, voltam ao palco os sistemas complementares de formação de memória e aprendizagem. Como já foi mencionado, o hipocampo auxilia na aprendizagem rápida de novas informações fonético-fonológicas. Esse conhecimento, além de ser reinstanciado no neocórtex, também pode ser usado antes que as novas formas-alvo sejam integradas no córtex, via monitoramento. A instrução explícita é imprescindível para que o monitoramento ocorra (ZIMMER e ALVES, no prelo), pois permite que o aprendiz faça uso do conhecimento processado no hipocampo, convertendo-o em produção oral. Essa conversão desempenha um papel relevante para que, além de meramente perceber, o aprendiz, através de repetidas exposições à forma-alvo, consiga notar as características distribucionais salientes no

${ }^{8}$ Isso se deve à capacidade que as redes conexionistas têm de processar tanto input ortogonal como input linearmente independente. Para o início de uma viagem fascinante rumo à compreensão dos mecanismos algébricos subjacentes aos algoritmos de aprendizagem utilizados em redes conexionistas, ver (nessa ordem): Jordan (1986), McClelland e Rumelhart (1988a), Rumelhart e McClelland (1986), McClelland e Rumelhart (1988b). 
insumo a que é exposto, levando à consolidação desse conhecimento. A noção de atenção, ligada à idéia de acesso a diferentes níveis de consciência, utilizada nas diferentes teorias de processamento do insumo no paradigma simbólico, está relacionada, no conexionismo, ao processo de consolidação, que é longo, gradual e contínuo.

\section{Contribuições e limitações dos paradigmas simbólico e conexionista para a aprendizagem de L2}

Pode-se sugerir que uma das contribuições feitas pelo paradigma simbólico à aprendizagem da L2 está, sobretudo, na área de estudos acerca do papel da atenção e da consciência e a relação dessas com a internalização, por parte do aprendiz, das estruturas léxico-gramaticais da L2. Uma outra contribuição é o debate teórico que decorre desses estudos, ou seja, aquele sobre a relação entre o conhecimento explícito e implícito na aprendizagem de L2 (HULSTIJN, 2005, p.134-135), o qual vem demonstrando que a formação de memórias de natureza explícita e implícita depende largamente do tipo de instrução e da estrutura em foco (ELLIS, 2005, p. 145-147). Outra contribuição está relacionada a uma melhor compreensão do papel da capacidade de memória de trabalho na retenção do conhecimento da L2, já que esse sistema cognitivo pode explicar diferenças individuais na aprendizagem.

Quanto às limitações, um dos principais problemas do paradigma simbólico relaciona-se justamente na dicotomia entre os conhecimentos implícito e explícito. Whittlesea e Wright (1997) afirmam que, enquanto a aprendizagem explícita é consciente e dinâmica, a implícita se caracteriza como estática e puramente desprovida de qualquer grau de consciência. Nesse sentido, Alves e Zimmer (2005) questionam como as regularidades dos sistemas lingüísticos seriam adquiridas implicitamente, se houvesse uma diferença tão grande entre os dois tipos de conhecimento. Whittlesea e Wright (1997) argumentam que a definição de aprendizagem implícita não se sustenta, tendo em vista que os sujeitos impõem organização sobre o estímulo através de um trabalho de organização tal que não precisa ser deliberado ou controlado conscientemente. A concepção simbólica de aprendizagem implícita vê o indivíduo como um sujeito passivo no processo de aprendizagem da L2. Dessa forma, a relação entre a consciência do aprendiz e a 
tradicional dicotomia implícito/explícito deve ser repensada, uma vez que o aprendizado implícito implica, também, atenção e manipulação dos dados do insumo por parte do aprendiz.

As contribuições feitas pelo paradigma conexionista à aprendizagem da L2 residem precisamente no fato de esse paradigma mudar o foco da estrutura para o processamento. Ou seja, em vez de enfatizar o papel de estruturas como a memória de trabalho na aprendizagem da L2, os pesquisadores conexionistas investigam como os aprendizes da L2 processam a informação lingüística, abordando conjuntamente o conhecimento lingüístico e o processamento do insumo da L2 e da L1 na pesquisa em L2. É com o modelo HipCort que se chega a uma explicação de cunho biológico sobre a natureza gradiente da interação entre os conhecimentos processados no hipocampo e no neocórtex, uma vez que o resultado do processamento rápido, que se dá no hipocampo, pode ser integrado gradualmente ao sistema do neocórtex. Esses dois sistemas, vistos como efetivamente complementares, conforme ressaltam McClelland et al. (1995), permitem que se desfaça a antinomia entre conhecimento implícito e explícito na aprendizagem da L2, além de oferecer uma explicação biologicamente mais plausível a questões como a fossilização, sugerindo alternativas de tratamento para ela, como a oferecida por McClelland (2001), já discutida na seção anterior.

As limitações do paradigma conexionista, principalmente levando-se em conta o contexto da Lingüística Aplicada, estão relacionadas ao número ainda pequeno de simulações conexionistas da aquisição da L2 feitas no exterior e no Brasil, que ainda não permitem avaliar o poder associativo da aprendizagem orientada pelo insumo. Atrelada a essa limitação, está a necessidade de especialização do pesquisador da L2 em áreas como a computação e a neurociência. Embora desejável e positiva, essa especialização exige tempo de formação e experiência com metodologias pouco utilizadas na Lingüística Aplicada, pelo menos no Brasil.

\section{Conclusão}

O objetivo deste trabalho foi abordar as pesquisas feitas em cognição e aprendizagem da L2 a partir dos diferentes pontos de vista acerca da cognição esposados pelos paradigmas simbólico e 
conexionista, discutindo como alguns construtos cognitivos são abordados em cada paradigma.

Percebeu-se, ao longo deste trabalho, que as noções de memória delineadas pelos diferentes paradigmas revelam a ênfase colocada na metáfora espacial pelo paradigma simbólico - que estipula a existência de diferentes espaços de armazenamento e processamento do insumo, como a memória de longo prazo, a memória de curto prazo e a memória de trabalho - e a diluição da distinção entre conhecimento e processamento propugnada pelo paradigma conexionista. No que tange ao processamento do insumo - serial no paradigma simbólico e simultâneo no conexionista -, a atenção desempenha um papel chave no processamento do insumo e na construção de estratégias metacognitivas, auxiliando o aprendiz a perceber e a manipular as formas-alvo de forma mais focal. Embora o papel da atenção seja bastante claro nas teorias de processamento do insumo no paradigma simbólico, seu conceito é mais difuso e menos conclusivo no que tange à plausibilidade biológica do acesso aos diferentes níveis de consciência, que também são mais seriais e estanques nesse paradigma; já o processamento implícito e o explícito do insumo são mais interativos e gradientes no paradigma conexionista.

É importante destacar que, a despeito das divergências sobre a natureza da cognição e dos construtos teóricos arroladas acima, os dois paradigmas apresentam visões convergentes no que diz respeito à importância do monitoramento e da instrução explícita na aquisição da L2, uma vez que, em ambos os paradigmas, as pesquisas vêm investigando o papel do professor na construção de uma consciência lingüística sintonizada tanto com as características prototípicas como com as idiossincráticas das formas-alvo.

Para finalizar, ressalta-se que, no estado atual da pesquisa em L2, qualquer tomada de posição com relação à escolha do "melhor" paradigma deve ser feita com cautela, uma vez que os resultados obtidos em estudos nos dois paradigmas, apesar de robustos, parecem ser inconclusivos quanto à explicação de como os seres humanos aprendem. É necessário, para um posicionamento definitivo, que sejam realizados mais estudos na área da aprendizagem de L2. 


\section{Referências Bibliográfias}

ALBANO, E. C. Criatividade e gradiência num léxico sem derivações. In: CABRAL, L. G.; MORAIS, J. (Ed.). Investigando a linguagem. Florianópolis: Mulheres, 1999. p. 35-54.

ALVES, U. K. O papel da instrução explicita na aquisição fonológica do inglês como L2: evidências fornecidas pela Teoria da Otimidade. 2004. 328f. Dissertação (Mestrado em Lingüística Aplicada), UCPel, Pelotas.

ALVES, Ubiratã K.; ZIMMER, Márcia C. Perceber, notar e aprender: uma visão conexionista da consciência do aprendiz na aquisição fonológica da L2. Revista Virtual de Estudos da Linguagem - ReVEL. Ano 3, n. 5, 2005. Disponível em: www.revelhp.cjb.net

ANDERSON, J. R.; LEBIERE, C.; LOVETT, M.C.; REDER, L. M. ACT-R: a higher-level account of processing capacity. In: Behavioral and Brain Sciences, v. 21, p. 831-52, 1998.

ASHCRAFT, M. H. Human memory and cognition. New York: Harper Collins, 1994.

ATKINSON, R.C.; SHIFFRIN, R. M. Human memory: a proposed system and its control processes. In: SPENCER, K. W. (Ed.). The psychology of learning and motivation: advances in research and theory. New York: Academic Press, 1968. p. 89-195.

BADDELEY, A. D. Short-term memory for word sequences as a function of acoustic, semantic, and formal similarity. Quarterly Journal of Experimental Psychology, v. 18, p. 362-365, 1966a.

The influence of acoustic and semantic similarity on long-term memory for word sequences. Quarterly Journal of Experimental Psychology, v. 18, p. 302-309, 1966b.

Working memory. Science, v. 255, p. 556-559, 1992.

Short-term and working memory. In: TULVING, E.; CRAIK, F. I. M. (Ed.). The Oxford handbook of memory. Oxford: Oxford University Press, 2000. p. 77-92.

BADDELEY, A. D.; HITCH, G. Working memory. In: BOWER, G. A. (Ed.). The psychology of learning and motivation: advances in research and theory. New York: Academic Press, 1974. p. 47-90. 
BADDELEY, A. D.; WARRINGTON, E. K. Amnesia and the distinction between long and short-term storage. Journal of Verbal Learning and Verbal Behavior, v. 9, p.176-189, 1970.

BATES, K.; MacWHINNEY, B. Functionalism and the competition model. In: MacWHINNEY, B.; BATES, K. (Eds.). The crosslinguistic study of language processing. Cambridge: Cambridge University Press, 1993. p. 3-73.

BERQUIST, B. Individual differences in working memory span and L2 proficiency: capacity or processing efficiency? Comunicação apresentada no Congresso Anual da Associação Americana de Lingüística Aplicada, Seattle, WA, 1998.

BOWER, G. H. A brief history of memory research. In: TULVING, E.; CRAIK, F. I. M. (Ed.). The Oxford handbook of memory. Oxford: Oxford University Press, 2000. p. 3-32.

BROEDER, P.; PLUNKETT, K. Connectionism and second language acquisition. In: ELLIS, N. (Ed.). Implicit and explicit learning. London: Academic, 1994. p. 421-454.

BROWN, J. Some tests of the decay theory of immediate memory. Quarterly Journal of Experimental Psychology, v. 10, p. 12-21, 1958.

CANTOR, J.; ENGLE, R. Working memory capacity as long-term activation: an individual differences approach. Journal of Experimental Psychology: Learning, Memory, and Cognition, v. 19, p.1101-1114, 1993.

CLARK, A. Microcognition: philosophy, cognitive science and parallel distributed processing. Oxford: Blackwell, 1989.

CONRAD, R.; HULL, A. J. Information, acoustic confusion and memory span. British Journal of Psychology, v. 55, p. 429-432, 1964.

DAMASIO, A. The feeling of what happens: body and emotion in the making of consciousness. New York: Harcourt, 2000.

ELLIS, N. Cognitive approaches to second language acquisition. Annual Review of Applied Linguistics, v. 19, p. 22-42, 1999.

. Memory for language. In: ROBINSON, P. (Ed.). Cognition and Second Language Acquisition. Cambridge: Cambridge University Press, 2001. p. 33-68.

. The study of second language acquisition. Oxford: OUP. 1994. 
ELLIS, N. Measuring implicit and explicit knowledge of a second language: a psychometric study. Studies in Second Language Acquisition, v. 27, p. 141-172, 2005.

ELMAN, J.; BATES, E.; JOHNSON, M.; KARMILOFF-SMITH, A.; PARISI, A.; PLUNKETT, K. Rethinking innateness: a connectionist perspective on development. Cambridge: M.A.: MIT, 1996.

ELMAN, J. Generalization from sparse input. Proceedings of the $38^{\text {th }}$ Annual Meeting of the Chicago Linguistics Society, 2003.

ENGLE, R. W., KANE, M. J.; TUHOLSKY, S. W. Individual differences in working memory capacity and what they tell us about controlled attention, general fluid intelligence, and functions of the prefrontal cortex. In: MIYAKE, A; SHAH, P. (Eds.). Models of working memory: mechanisms of active maintenance and executive control. Cambridge: Cambridge University Press, 1999. p. 102-134.

ERICSSON, K. A.; KINTSCH, W. Long-term working memory. Psychological Review, v. 102, p. 211-245, 1995.

EYSENCK, M. W. (Ed.). The Blackwell dictionary of cognitive psychology. Cambridge, MA: Blackwell, 1994.

FLEGE, J. E. Interactions between the native and second-language phonetic systems. In: BURMEISTER, P.; PIRSKE, T.; RHODE, A. An integrated view of language development: papers in honor of Henning Wode. Trier: Wissenschaftliger Verlag, 2002. p. 217-243.

FLEGE, J. E.; LIU, S. The effect of experience on adults' acquisition of a second language. Studies on Second Language Acquisition, v. 23, p. 527-552, 2001.

FODOR, J.; PYLYSHYN, Z. Connectionism and cognitive architecture: a critical analysis. Cognition, v. 28, p. 3-71, 1988.

FORTKAMP, M. B. M. Working memory capacity and aspects of L2 speech production. Communication and Cognition, v. 32, p. 259-296, 1999.

. Working memory capacity and L2 speech production: an exploratory study. 2000. 230f. Tese (Doutorado em Inglês e Lingüística Aplicada) Universidade Federal de Santa Catarina, Florianópolis.

. Working memory capacity and fluency, accuracy, complexity, and lexical density in L2 speech production. Fragmentos, no prelo. 
GASSER, M. Connectionism and universals of second language acquisition. Studies on Second Language Acquisition, v. 12, p. 179-199, 1990.

GRAF, P.; SCHACTER, D.L. Implicit and explicit memory for new association in normal and amnesic subjects. Journal of Experimental Psychology: Learning, Memory, and Cognition, v. 11, p. 501-518, 1985.

. Selective effects of interference on implicit and explicit memory for new associations. Journal of Experimental Psychology: Learning, Memory, and Cognition, v. 13, p. 45-53, 1987.

GOPHER, D. Attention: phenomenology, history, and scientific perspective. In: EYSENCK, M.W. (Ed.). The Blackwell dictionary of cognitive psychology. Cambridge, MA: Blackwell, 1994, p. 23-28.

HABERLANDT, K. Cognitive Psychology. Massachusetts: Allyn and Bacon, 1994.

HARRINGTON, M. Working memory capacity as a constraint on L2 development. In: HARRIS, R.J. (Ed.). Cognitive processing in bilinguals. Amsterdam: Elsevier, 1992. p. 123-135.

HARRINGTON, M.; SAWYER, M. L2 working memory capacity and L2 reading skill. Studies in Second Language Acquisition, v. 14, p. 25-38, 1992.

HAYKIN, S. Neural networks. Ontario: Macmillan College, 1994.

HULSTIJN, J. H. Theoretical and empirical issues in the study of implicit and explicit second-language learning. Studies in Second Language Acquisition, v. 27, p. 129-140, 2005.

IZUMI, S.; BIGELOW, M. Does output promote noticing and second language acquisition? TESOL Quarterly, v. 34, p. 239-278, 2000.

IZUMI, S. Output, input enhancement, and the noticing hypothesis: An experimental study on ESL relativization. Studies in Second Language Acquisition, v. 24, p. 541-577, 2002.

JORDAN, M. I. An introduction to linear algebra in parallel distributed processing. In: RUMELHART, D.; McClELlAND, J. (Ed.). Parallel distributed processing: explorations in the microstructure of cognition. Cambridge, MA: MIT, v. 1, 1986. p. 365-422.

KANDEL, E.; SCHWARTZ, J.; JESSEL, T. Essentials of neural science and behavior. Englewood Cliffs, NJ: Prentice Hall, 1995. 
KEMPE, V.; MacWHINNEY, B. Acquisition of case marking by adult learners of German and Russian. Studies on Second Language Acquisition, v. 3, p. 114-138, 1999.

KRASHEN, S. Second language acquisition and second language learning. Oxford: Pergamon Press, 1982.

LEAHEY, T. A bistory of psychology: Main currents in psychological thought. Englewood Cliffs, NJ: Prentice Hall, 1987.

LEOW, R. P. Attention, awareness, and foreign language behavior. Language Learning, v. 47, p. 467-505, 1997.

MacDONALD, M.; CHRISTIANSEN, M. Reassessing working memory: a reply to Just \& Carpenter and Waters \& Caplan. Psychological Review, v. 109, n. 1, p. 35-54, 2002.

MacWHINNEY, B. Transfer and competition in second language learning. In: HARRIS, R. (Ed.). Cognitive processing in bilinguals. Amsterdam: North Holland, 1992. p. 57-83.

. Second language acquisition and the competition model. In: GROOT, A. M. B.; KROLL, J. F. (Ed.). Tutorials in bilingualism: psycholinguistic perspectives. Mahwah: Erlbaum, 1997. p. 103-139.

The competition model: the input, the context, and the brain. In: ROBINSON, P. (Ed.). Cognition and second language instruction. Cambridge: CUP, 2001. p. 69-90.

Language Emergence. In: BURMEISTER, P., PISKE, T.; RHODE, A. (Ed.). An integrated view of language development. Papers in bonor of Henning Wode. Trier: Wissenchaftliche Verlag, 2002. p. 17-42.

McCLELLAND, J. L. Failures to learn and their remediation: a Hebbian account. In: McCLELLAND, J. L.; SIEGLER, R. S. (Ed.). Mechanisms of cognitive development: behavioral and neural perspectives. Mahwah: Lawrence Erlbaum Associates, 2001. p. 97-121.

McCLELLAND, J. L.; RUMELHART, D. E. Learning in PDP models: the pattern associator. In: (Ed.). Explorations in Parallel Distributed Processing: a handbook of models, programs, and exercises. Cambridge, MA: MIT Press, 1988a. p. 83-120.

. Training hidden units: the generalized delta rule. In:

Explorations in Parallel Distributed Processing: a handbook of models, programs, and exercises. Cambridge, MA: MIT Press, 1988b. p. 121-159. 
McClELLAND, J. McNAUGHTON, B.; O'REILY, R. Why there are complementary learning systems in the hippocampus and neocortex: insights from the successes and failures of connectionist models of learning and memory. Psychological Review, v.102, n.103, p. 419-457, 1995.

MENDONÇA, D. M. Working memory capacity and the retention of $L 2$ vocabulary. 2003. 101f. Dissertação (Mestrado em Letras/Inglês e Literatura Correspondente) - Universidade Federal de Santa Catarina, Florianópolis. MINSKY, M. A framework for representing knowledge. In: HAUGELAND, J. (Ed.). Mind Design. Cambridge, MA.: MIT., 1981.p. 332-356.

MIYAKE, A.; FRIEDMAN, N. P. Individual differences in second language proficiency: working memory as language aptitude. In: HEALY, A. F.; BOURNE, L. E. Jr. (Ed.). Foreign language learning: psycholinguistic studies on training and retention. Mahwah, NJ: Lawrence Erlbaum, 1998. p. 339-364.

MIYAKE, A.; SHAH. A. Toward unified theories of working memory: emerging general consensus, unresolved theoretical issues, and future research directions. In: MIYAKE, A.; SHAH, P. (Ed.). Models of working memory: mechanisms of active maintenance and executive control. Cambridge: Cambridge University Press, 1999, p. 442-481.

NEWELL, A. Physical symbol systems. Cognitive Science, v. 4, p. 135-83, 1980.

NEWELL, A.; ROSENBLOOM, P. S.; LAIRD, J. E. Symbolic architectures for cognition. In: POSNER, M. (Ed.). Foundations of cognitive science. Cambridge, MA: MIT, 1998. p. 436-459.

PLAUT, D. C. What sort of computation is cognition? Palestra ministrada no PDP meeting, Carnegie Mellon University, 15 jan. 2003.

PlAUT, D. C., McClEllAND, J., SEIDENBERG, M.; PATTERSON, K. Understanding normal and impaired word reading: computational principles in quasi-regular domains. Psychological Review, n.103, p. 56-115, 1996.

PETERSON, L. R; PETERSON, M. J. Short-term retention of individual verbal items. Journal of Experimental Psychology, v. 58, p. 193-198, 1959.

RHODE, D. L. T.; PLAUT, D. C. Connectionist models of language processing. Cognitive Studies, v.10, p.10-28, 2003.

RINALDI, V. Conexionismo e aquisição de lingua estrangeira. Comunicação apresentada no 6을 Encontro Nacional sobre Aquisição da Linguagem, Porto Alegre, RS, 2003. 
RINALDI, V.; FRANÇOZO, E. Um modelo conexionista para a memória bilingüe. Comunicação apresentada no XIX Encontro Nacional da ANPOLL, Maceió, AL, 2004.

ROBINSON, P. Cognition and second language instruction. Cambridge: Cambridge University Press, 2001. 453p.

RUMELHART, D. Learning internal representations by error propagation. In: RUMELHART, D.; McCLELLAND, J. (Ed.). Parallel distributed processing: explorations in the microstructure of cognition. Cambridge, Mass: MIT, v. 2, 1986. p. 318-362.

RUMELHART, D.; McCLELLAND, J. (Ed.). Parallel distributed processing: explorations in the microstructure of cognition. Cambridge, Mass: MIT, v. 2, 1986. p. 496p.

RYLE, G. The concept of mind. London: Hutchinson, 1949.

SAFFRAN, J. R. Words in a sea of sounds: the output of statistical learning. Cognition, v. 81, p. 149-169, 2001.

SCHACTER, D. L. Implicit memory: history and current status. Journal of Experimental Psychology: Learning, Memory, and Cognition, v. 13, p. 501518, 1987.

SCHMIDT, R. The role of consciousness in second language learning. Applied Linguistics, v. 11, p. 129-158, 1990.

SCHMIDT, R. Consciousness and foreign language learning: A tutorial on the role of attention and awareness in learning. In: SCHMIDT, R. (Ed.). Attention and awareness in foreign language learning (Technical Report \# 9). Honolulu, Hawaii: University of Hawaii, Second Language Teaching \& Curriculum Center, 1995. p. 1-63.

SCHMIDT, R. Attention. In: ROBINSON, P. (Ed.). Cognition and second language instruction. Cambridge: Cambridge University Press, 2001. p. 3-32.

SCHUMANN, J. Extending the scope of the acculturation/pidgnization model to include cognition. TESOL Quarterly, v. 24, p. 667-683, 1990.

SEIDENBERG, M. S; MacDONALD, M. A probabilistic constraints approach to language acquisition and processing. Cognitive Science, 23, p. 569-588, 1999.

SEIDENBERG, M. S; MacDONALD, M.; SAFFRAN, J. R. Does grammar start where statistics stop? Science, v. 218, p. 553-554, 2002. 
SHANKS, D. Breaking Chomsky's rules. New Scientist, v. 137, p. 26-30, 1993. SHAW, P.; MIYAKE, A. Models of working memory: an introduction. In: MIYAKE, A.; SHAH, P. (Ed.). Models of working memory: mechanisms of active maintenance and executive control. Cambridge: Cambridge University Press, 1999, p. 1-27.

SIGOT, A.E. The processing of inferences in reading in English as a foreign language according to a connectionist approach. 2002. 159f. Tese (Doutorado em Lingüística Aplicada) - Pontifícia Universidade Católica do Rio Grande do Sul, Porto Alegre.

SOKOLIK, M. E. Learning without rules: PDP and a resolution of the adult language learning paradox. TESOL Quarterly, v, 24, n. 4, p. 685-696, 1990.

SOUGNÉ, J.; FRENCH, R. M. A Neurobiologically inspired model of working memory based on neuronal synchrony and rythmicity. In: BULLINARIA, J. A.; GLASSPOOL, D. W.; HOUGHTON, G. (Eds.). Proceedings of the Fourth Neural Computation and Psychology Workshop: Connectionist Representations. London: Springer-Verlag, 1997, p. 155-167.

STERNBERG, R. J. Cognitive Psychology. Belmont: Wadsworth/Thomson Learning, 2003.

SWAIN, M. Three functions of output in second language learning. In COOK, G.; SEIDLHOFER, B. (Eds.). Principle E practice in applied linguistics: studies in honor of H.G. Widdowson. Oxford: Oxford University Press, 1995. p. 125144 .

SWAIN, M.; LAPKIN, S. Problems in output and the cognitive processes they generate: a step towards second language learning. Applied Linguistics, v. 16, p. 371-391, 1994.

SWAIN, M. Communicative competence: some roles of comprehensible input and comprehensible output in its development. In: GASS, S; MADDEN, C. (Eds.). Input in second language acquisition. Rowley, MA: Newbury House, 1985. p. 235-253.

TORRES, A. C. G. Working memory capacity and readers' performance on main idea construction in L1 and L2. 2003. 215f. Tese (Doutorado em Inglês e Lingüística Aplicada) - Universidade Federal de Santa Catarina, Florianópolis.

TORSUN, I. S. Foundations of intelligent knowledge-based systems. London: Academic Press, 1995. 402p. 
TULVING, E. Episodic and semantic memory. In: TULVING, E; DONALDSON, W. (Ed.). Organization of memory. New York: Academic Press, 1972. p. 381-403.

. How many memory systems are there? American Psychologist, v. 40, p. 385-398, 1985.

Concepts of memory. In: TULVING, E; CRAIK, F. I. M. (Eds.). The Oxford handbook of memory. New York: Oxford University Press, 2000. p. 33-43.

VanPATTEN, B. Attending to form and content in the input: an experiment in consciousness. Studies in Second Language Learning, v. 12, p. 287-301, 1990.

Input processing and grammar instruction in second language acquisition. Norwood, N. J. Ablex, 1996.

. Procesing instruction: theory, research, and commentary. Mahwah:

Lawrence Erlbaum, 2004.

VanPATTEN, B.; CADIERNO, T. Explicit instruction and input processing. Studies in Second Language Acquisition, v.15, p. 225-243, 1993.

VanPATTEN, B.; SANZ, C. From input to output: processing instruction and communicative tasks. In: ECKMAN, F.R.; HIGHLAND, P.W.; MILEHAM, J. WEBER, R. R. (Eds.). Second language acquisition theory and pedagogy. Mahwah, NJ: Lawrence Erlbaum, 1995. p. 169-185.

WHITTLESEA, B. W. A.; WRIGHT, R. L. Implicit (and explicit) learning: acting adaptively without knowing the consequences. Journal of experimental Psychology: Learning, Memory, and Cognition, v. 23, n.1, p. 181-200, 1997.

ZIMMER, M. C. A transferência do conbecimento fonético-fonológico do PB (L1) para o inglês (L2) na recodificação leitora: uma abordagem conexionista. 2004a. 187f. Tese (Doutorado em Lingüística Aplicada) Pontifícia Universidade Católica do Rio Grande do Sul, Porto Alegre.

O conexionismo e a leitura de palavras. In: ROSSA. A. e ROSSA, C. Rumo à psicolingüística conexionista. Porto Alegre: EDIPUCRS, 2004b. p.101-138.

ZIMMER, M. C.; ALVES, U. K. A produção de aspectos fonéticos/fonológicos da L2: instrução explícita e conexionismo. Linguagem e ensino, no prelo. 